\title{
1 Supplementation of a lacto-fermented rapeseed-seaweed blend 2 promotes gut microbial- and gut immune-modulation in weaner 3 piglets
}

$4{ }^{1}$ Yan Hui, ${ }^{2}$ Paulina Tamez-Hidalgo, ${ }^{1}$ Tomasz Cieplak, ${ }^{3}$ Gizaw Dabessa Satessa, ${ }^{4}$ Witold Kot,

$5 \quad{ }^{2}$ Søren Kjærulff Søren, ${ }^{5}$ Mette Olaf Nielsen, ${ }^{1}$ Dennis Sandris Nielsen, ${ }^{1 *}$ Lukasz Krych

$6{ }^{1}$ Department of Food Science, Faculty of Science, University of Copenhagen. Rolighedsvej 26, DK71958 Frederiksberg C, Denmark.

$8 \quad{ }^{2}$ Fermentationexperts A/S. Vorbassevej 12, DK-6622 Bække, Denmark.

$9{ }^{3}$ Department of Veterinary and Animal Sciences, Faculty of Health and Medical Sciences, 10 University of Copenhagen. Grønnega $\square$ rdsvej 3, DK-1870 Frederiksberg C, Denmark.

$11{ }^{4}$ Department of Plant and Environmental Sciences, Faculty of Science, University of Copenhagen. 12 Rolihedsvej 26, DK-1958 Frederiksberg C, Denmark.

$13{ }^{5}$ Department of Animal Science, Faculty of Technical Sciences, Aarhus University. Blichers Allé 14 20, DK-8830 Tjele, Denmark.

$15 *$ Corresponding Author

16 Corresponding Address:

17 krych@food.ku.dk 


\section{Abstract}

The direct use of medical zinc oxide $(\mathrm{ZnO})$ in feed will be abandoned after 2022 in Europe, leaving an urgent need for substitutes to prevent post-weaning disorders. This study assessed whether rapeseed meal added two brown macroalagae species (Saccharina latissima and Ascophylum nodosum) and fermented using lactic acid bacteria (FRS) could improve piglet performance and gut health. The weaned piglets were fed one of three different feeding regimens ( $\mathrm{n}=230 \mathrm{each}$ ): basal diet, $2.5 \%$ and $5 \%$ FRS from day 28 of life to day 85 . The piglets fed with $2.5 \%$ FRS presented superior phenotype with alleviated intraepithelial and stromal lymphocytes infiltration in the gut, enhanced colon mucosa barrier as well as numerically improvements of final body weight. Colon microbiota composition was determined using amplicon sequencing of the V3 and V1 - V8 region of the 16S rRNA gene using Illumina Nextseq and Oxford Nanopore MinION sequencing, respectively. The two amplicon sequencing strategies showed high consistence between the detected bacteria. Both sequencing technologies showed that the FRS fed piglets had a distinctly different microbial composition relative to the basal diet. Compared with piglets fed the basal diet, Prevotella stercorea was verified by both technologies to be more abundant in the FRS piglets, and positively correlated with colon mucosa thickness and negatively correlated with blood levels of leucocytes and IgG. In conclusion, FRS supplementation improved gut health of weaner piglets, and altered their gut microbiota composition. Increasing the dietary inclusion of FRS from $2.5 \%$ to $5 \%$ did not cause further improvements.

\section{Keywords}




\section{Introduction}

A healthy hindgut is essential for the optimal nutrient utilization and health of the host. Essentially, bacteria largely ferment and digest macronutrients such as insoluble fibers and undigested proteins in the colon (Zeng 2014) (Jha and Berrocoso 2016). Bacterial metabolism benefits colonic cells by providing butyrate and the immune system by providing acetate and propionate (Zeng 2014). However, the process of bacterial colonization, which brings benefits for the host, also brings the risk of infection-inflammation responses. Thus, bacterial association and communication with the immune system balances on a thin line for preserving homeostasis (Peterson and Artis 2014) between the learning of what to attack and what to tolerate (Scudellari 2017).

In pig production, the weaning period is characterized by a change in diet from milk to solid feed, separation from the mother and aggregation in a pen with piglets from other litters. It is the most stressful period in a pig's life and often lead to morbidities like diarrhea. In-feed zinc-oxide used to be a prevalent choice for prophylaxis, but according to the European Union regulations, zinc-oxide shall no longer be directly used in feed or water from 2022 (Satessa et al. 2020a). This leaves an urgent need for new prophylactic substitutes. Additive inclusion of pre-fermented feeds has been regarded as a promising strategy to ameliorate these post-weaning disorder issues for its effective act to improve gastrointestinal health and enhance livestock performance in production (Hu et al. 2008) (Heres et al. 2003) (Wang et al. 2018). The process of microbial fermentation degrades antinutritional compounds and macronutrients in feed, which increases the nutrient bioavailability and nutritional value (Canibe et al. 2007) (Mukherjee et al. 2015). Besides, the dominant microorganisms in fermented feed have been proposed to inhibit the overgrowth of opportunistic pathogens thus sustaining gut microbiome balance (Plumed-Ferrer and von Wright 2009) and thereby promote immune functions of the host (Zhou et al. 2015). A meta-analysis has shown that fermented feed is able to boost the growth and performance of both weaner and growing pigs (Xu et al. 2020). Supplementation of fermented rapeseed meal also improves the intestinal morphology of broiler chickens (Hu et al. 2016).

Rapeseed meal is a by-product after the oil has been extracted and is generally used as a protein source in animal diets (van der Spiegel et al. 2013). However, it has a lower protein digestibility as compared to soybean meal. Brown seaweed is acknowledged as a good source of health-promoting phytochemicals (Cherry et al. 2019) with bioactive compounds like laminarin (Zargarzadeh et al. 2020), but it has poor digestibility as well. The fermentation with lactic acid bacteria of a rapeseed- 
73 seaweed (FRS) blend could improve the health and nutritional value by reducing the naturally 74 present anti-nutritional factors and by hydrolyzing protein and fibers down to a more soluble 75 matrix. We have previously reported that dietary supplementation with FRS could contribute 76 favorably in the development of a healthy functional gut barrier as well as the production 77 performance of piglets in the weaning period (Satessa et al. 2020a).

78 In the present study, we tested whether addition of FRS to weaner diets will affect modulation of 79 the gut microbiome and the immune function. We chose weaning as the physiological stress 80 situation to investigate this process and to learn how dietary induced gut modulation translates into 81 body weight gain and gut health or illness later in life. To study gut microbial composition in 82 response to dietary intervention, we have used two sequencing strategies of 16S rRNA gene 83 amplicons and compared their performance: 2nd generation (Illumina, NextSeq) and 3rd generation 84 (Oxford Nanopore Technologies, MinION). 


\section{Materials and Methods}

\section{Preparation of fermented rapeseed meal-seaweed feed}

The fermented rapeseed meal-seaweed (FRS) feed was provided by FermentationExperts (Denmark), which was a blend of rapeseed meal (Brassica napus), wheat bran (Triticum eastivum) and two types of brown seaweed (Saccharina latissima and Ascophylum nodosum) prepared via a controlled 2-step solid state fermentation. The inoculum consists of three lactic acid bacteria: Pediococcus acidilactici (DSM 16243), Pediococcus pentosaceus (DSM 12834) and Lactobacillus plantarum (DSM 12837). The addition of the inoculant controlled the process by acidifying the blend within the first 24 hours, and assuring an almost entirely anaerobic process. The process continued for 11 days at $38{ }^{\circ} \mathrm{C}$. The fermented material was then dried in a spin flash dryer, with a temperature setting and pass-through-speed that preserved the viable bacteria and the microbial thermolabile metabolites.

\section{Feeding and recording of piglet performance}

This study was a feeding trial carried out on a commercial pig farm (Kawiks Farm, Patoki 23. 98170 Widawa. Province. Lodz city, Poland) in 2018, where groups of piglets were weaned one day a week over a 5-week period. The trial procedure and sample collection were approved by the Local Ethical Commission of Olsztyn University of Life Sciences (Olsztyn, Poland) with respect to experimentation and care of animals under study. A total of 690 piglets were tested under three different feeding regimens (230 piglets per feeding treatment) from 28 days of age (10 days before weaning) until 85 days of age when piglets exited the nursing unit. One group was a control group fed a basal feed according to Danish nutritional recommendations (Tybirk 2015), and the other two groups received supplementation of $2.5 \%$ or $5 \%$ FRS to the basal feed (feed dry matter basis) (Table 1). Piglets on each dietary regime were housed in nursing pens holding an average of 48 animals per pen. Each dietary treatment was repeated 5 times (1 repetition per experimental week and 1 pen representing a repetition) and the control was repeated 4 times. None of the diets included growth promoters, prescription antibiotics or zinc oxide. Piglets that experienced diarrhea or any other serious health conditions were removed from the experiment and treated elsewhere and counted as piglets that did not complete the experiment. Feed and fresh water were supplied $a d$ libitum throughout the experiment. 
115 Litter weights during the nursing period were recorded every week, and feed intake was recorded

116 daily. Performance indicators such as body weight, ADFI (average daily feed intake), average daily

117 weight gain (ADG), feed conversion ratio (FCR) and completion rate were calculated at the pen

118 level as previously outlined (Satessa et al. 2020a).

\section{Biological sample collection}

120 A total of 10 piglets from each treatment (5 in each of two experimental weeks) were randomly

121 selected and euthanized 3 weeks after weaning. The animals were euthanized by stun gunning with

122 a captive bolt immediately followed by de-bleeding at the farm slaughtering facilities under strict

123 sanitary regulations. Whole blood samples and serum for clinical analysis, the digesta from the

124 colon for microbiome analysis, and jejunum and colon tissues for histopathological analyses, were

125 collected in that order immediately after slaughtering.

126 A blood sample from each piglet was deposited in a tube with the anti-coagulant EDTA and 127 preserved on ice until taken to the laboratory, where it was stored at $2-8{ }^{\circ} \mathrm{C}$ until analysis. Another 128 blood sample was collected in a tube without anticoagulant, and serum separated by centrifugation, 129 which was then stored at $-20{ }^{\circ} \mathrm{C}$ until analysis. Gut tissues and colon contents were sampled after 130 opening of the abdominal wall, and the stomach, small and large intestines were occluded at both 131 ends and removed. Approximately $2 \mathrm{~cm}^{3}$ of colon content was collected from the apex of the 132 ascending spiral of the colon with a sterile spatula and deposited in cryotubes with RNAlater ${ }^{\mathrm{TM}}$ 133 (Sigma-Aldrich, Munich, Germany). Tubes with colon contents were kept at room temperature for 134 less than $24 \mathrm{~h}$, followed by cryopreservation in the laboratory. Tissue samples (approximate $2 \mathrm{~cm}$ 135 long) of the whole transection of the jejunum and colon were excised and carefully rinsed from gut 136 contents by flushing with saline $(0.9 \% \mathrm{NaCl})$. For each tissue a sterilized blade was used. Tissues 137 were preserved in $10 \%$ formaldehyde and kept at room temperature for no longer than 24 hours 138 until further processing (Satessa et al. 2020b).

\section{Blood hematology, blood biochemistry and serum immunoglobulin analysis}

140 Full blood counts (erythrocyte, hemoglobin, hematocrit, mean corpuscular volume, mean 141 corpuscular hemoglobin, mean corpuscular hemoglobin concentration, red cell distribution width) 142 and differential white blood cells count (platelets, leucocytes, lymphocytes, monocytes, neutrophils, 143 eosinophils, basophils) were performed using a Sysmex XT 2000i analyzer (Sysmex Corporation, 
144 Kobe, Japan). Serum analysis measured concentrations of the following, using standardized

145 quantification methods: alanine aminotransferase, glutamic pyruvic transaminase, aspartate

146 aminotransferase, glutamic-oxaloacetic transaminase, lactate dehydrogenase, lysozyme, glucose,

147 total protein, blood urea nitrogen, uric acid, phosphorous, total cholesterol, triglycerides, low

148 density lipoprotein, high density lipoprotein and immunoglobulin $\mathrm{G}$ (IgG) according to previously

149 described procedures (Satessa et al. 2020a).

\section{Histological morphometric analysis of intestinal tissues}

151 The histological analysis of mid-jejunal and colonic tissues was conducted by a commercial 152 analytical laboratory (ALAB Weterynaria, Warsaw, Poland) according to previously described 153 procedures (Satessa et al. 2020a). In short, tissue sections fixed in 10\% formaldehyde were 154 dehydrated by means of graded ethanol and xylene baths and embedded in paraffin wax, and 3-4 $155 \mu \mathrm{m}$ section were then stained with haematoxylin and eosin. Histopathological evaluations (at 156 different lens magnifications) measured gut-associated lymphoid tissue (GALT), intraepithelial 157 lymphocytes (IELs) and lymphatic infiltration of the stromal mucosa (stromal lymphocytes, SL) 158 counts. In the GALT scoring, the numbers of lymphoid follicles per millimeter square were 159 counted. For IEL scoring, the following scale was used: 0-normal (0-10 IELs/100 enterocytes), 1160 low (10-15 IELs/100 enterocytes), 2-moderate (15-20 IELs/100 enterocytes; this level suggests 161 chronic subclinical inflammation, where the intestinal-blood barrier may be damaged; weak 162 lymphocytic inflammation), 3-severe (> 20 IELs/100 enterocytes; this level indicates chronic 163 inflammation with infiltration damaging the epithelium and intestinal-blood barrier; moderate 164 lymphocytic inflammation). For SL, the visual scoring scale was: 0-normal (single lymphocytes in 165 stromal connective tissues of villus and crypts), 1-low (increased number of lymphocytes, but no 166 damage to the stroma structures), 2-moderate (abundant infiltration of lymphocytes in stroma, 167 damaging blood vessel walls, connective tissue fiber, reducing visibility of stroma structures), 3168 severe (lymphocyte infiltration completely disrupts and conceals the stroma). In a blinded fashion, 16910 fields of view per piglet at $4 \times$ magnification were used for evaluation of GALT structures and 170 numbers of lymphoid follicles. IEL and SL were evaluated at 40× magnification. The analysis used 171 a standard light microscope Olympus BX41 and Cell Sens software (Olympus Corporation, Tokyo, 172 Japan). The gut tissues samples which could not reach the requirements for histological analysis 173 were discarded, which resulted in 9, 8, 10 piglets included in analysis for the basal diet, 2.5\% and $1745 \%$ FRS groups, respectively. 


\section{$17516 \mathrm{~S}$ rRNA gene amplicon sequencing of colon content}

176 Collected colon contents was stored at $-60{ }^{\circ} \mathrm{C}$ prior to the analysis. Two types of $16 \mathrm{~S}$ rRNA gene 177 amplicon sequencing strategies were adopted to characterize the prokaryotic community: Illumina, 178 NextSeq (Illumina, CA, USA) and MinION (Oxford Nanopore Technologies, Oxford, UK). The 179 genomic DNA was extracted using Bead-Beat Micro AX Gravity Kit (A\&A Biotechnology, 180 Gdynia, Poland) according to the manufacturer's instruction. DNA concentration and purity were 181 measured using NanoDrop ND-1000 spectrophotometer (Saveen and Werner AB, Sweden).

182 Extracted DNA was diluted to $10 \mathrm{ng} / \mathrm{\mu L}$ prior to library preparation. The $\mathrm{V} 3$ hypervariable region 183 of 16S rRNA gene was amplified and sequenced with Illumina technology as previously described 184 (Krych et al. 2018). Near full-length 16S rRNA gene amplicons were amplified and sequenced with 185 ONT targeting V1-V8 hypervariable region using following primers: ONT_27Fa: GTCTCGTGGG 186 CTCGGAGATG TGTATATAGA TCGCAGAGTT TGATYMTGGCTCAG; ONT_27Fb: 187 GTCTCGTGGG CTCGGAGATG TGTATATAGA TCGCAGAGTT TGATCCTGGCTTAG and ONT_1540_R: GTCTCGTGGG CTCGGAGATG TGTATACTCT CTATTACGGY TACCTTGTTACGACT. Custom designed barcoding system was developed to tag encode up to 96 samples during the second round of PCR, and the PCR primer sequence is given in Appendix Table 1. The PCR1 reaction mix contained $5 \mu$ of PCRBIO buffer and $0.25 \mu \mathrm{L}$ PCRBIO HiFi polymerase

192 (PCR Biosystems Ltd, London, United Kingdom), $1 \mu \mathrm{L}$ of primers mix (5 $\mu \mathrm{M}$ of ONT_27Fa and 193 ONT_27Fb, and $10 \mu \mathrm{M}$ of ONT_1540_R, see above), $5 \mu \mathrm{L}$ of genomic DNA ( 10ng/ $\mu \mathrm{L})$ and 194 nuclease-free water to a total value of $25 \mu \mathrm{L}$. The PCR thermal conditions were as follows: 195 denaturation at $95^{\circ} \mathrm{C}$ for $5 \mathrm{~min} ; 33$ cycles of $95^{\circ} \mathrm{C}$ for $20 \mathrm{~s}, 55^{\circ} \mathrm{C}$ for $20 \mathrm{~s}$ and $72^{\circ} \mathrm{C}$ for $45 \mathrm{~s}$; 196 followed by final elongation at $72^{\circ} \mathrm{C}$ for $4 \mathrm{~min}$.

197 PCR products were verified by agarose gel electrophoresis and then subjected for barcoding 198 (PCR2). The PCR2 mix composed of $5 \mu \mathrm{L}$ PCRBIO buffer, $0.25 \mu \mathrm{L}$ PCRBIO HiFi polymerase (PCR Biosystems Ltd, London, United Kingdom), $2 \mu \mathrm{L}$ of barcode primers $(5 \mu \mathrm{M})$ (Appendix 200 Table 1) $1 \mu \mathrm{L}$ of PCR1 template and DEPC water up to $25 \mu \mathrm{L}$. The PCR2 thermal conditions were 201 as follows: denaturation at $95{ }^{\circ} \mathrm{C}$ for 2 mins; 13 cycles of $95{ }^{\circ} \mathrm{C}$ for $20 \mathrm{~s}, 55^{\circ} \mathrm{C}$ for $20 \mathrm{~s}, 72{ }^{\circ} \mathrm{C}$ for $20240 \mathrm{~s}$; final elongation at $72{ }^{\circ} \mathrm{C}$ for 4 mins. The final PCR products were purified using AMPure XP 203 beads (Beckman Coulter Genomic, CA, USA) and pooled in equimolar concentrations. The pooled 204 barcoded amplicons were subjected to 1D genomic DNA by ligation protocol (SQK-LSK109) to 205 complete library preparation for MinION sequencing. Approximate $0.2 \mu \mathrm{g}$ of amplicons were used 
206 for the initial step of end-prep. And $40 \mathrm{ng}$ of prepared amplicon library was loaded on a R9.4.1 flow 207 cell.

208 Sequencing data analysis

209 The raw Illumina derived dataset containing pair-ended reads with corresponding quality scores 210 were merged and trimmed using fastq_mergepairs and fastq_filter scripts implemented in the 211 USEARCH pipeline as described previously (Krych et al. 2018). Purging the dataset from chimeric 212 reads and constructing zero radius Operational Taxonomic Units (zOTU) was conducted using the 213 UNOISE. The Greengenes (13.8) 16S rRNA gene collection was used as a reference database 214 (DeSantis et al. 2006).

215 Data generated by MinION were collected using MinKnow software v19.06.8 216 (https://nanoporetech.com). The Guppy v3.2.2 basecalling toolkit was used to base call raw fast5 to 217 fastq (https://nanoporetech.com). Porechop v0.2.2 was used for adapter trimming and sample 218 demultiplexing (https://github.com/rrwick/Porechop). The Porechop adapter list was (adapters.py) 219 edited accordingly and is given in Appendix Table 1. Sequences containing quality scores (fastq 220 files) were quality corrected using NanoFilt ( $q \geq 10$; read length $>1 \mathrm{~Kb}$ ). Taxonomy assignment of 221 quality corrected reads against Greengenes (13.8) database was conducted using uclast method 222 implemented in parallel_assign_taxonomy_uclust.py QIIME (v1.9.1). The uclust settings were 223 tuned on mock communities (--similarity 0.8; min_consensus_fraction 0.51) assuring annotations to 224 the lowest taxonomic level with no false positive annotations. The settings allowed it to treat 225 individual Oxford Nanopore Technologies - Amplicon Sequence Variant (ONT-ASV) as individual 226 "seeds". Reads classified to at least phylum level were subjected for further analysis.

\section{Statistics}

228 All the statistical analysis concerning phenotype data was performed with $\mathrm{R}$ (v3.6.2). The 229 difference of piglets performance on the pen level was evaluated using linear mixed model as 230 previously outlined (Satessa et al. 2020a) and orthogonal polynomial contrast was used to 231 appreciate the effect on increasing dose of the FRS $(0 \%, 2.5 \%, 5 \%)$. For histology and hematology 232 data, Wilcoxon rank test was used to evaluate statistical significance between different treatment 233 groups. 
234 For microbiome analysis, QIIME2 (Bolyen et al. 2019) (v2018.11) combined with R packages

235 (ggplot2, vegan, corrplot, Rhea, rstatix, vennDiagram) were used. Three samples were removed due 236 to inadequate library size (<1000 counts), which resulted in 9, 8, 10 piglets included for basal diet, $2372.5 \%$ and 5\% FRS group, respectively. Considering both types of amplicon profiling, all the 238 samples were summarized at the L7 levels (species) and rarefied to the same sequencing depth 239 (11000 reads/sample) for alpha and beta diversity calculations on both platforms. The standard 240 rarefaction at ASV level was conducted on Illumina data as comparison for rarefaction on species 241 level. Principal coordinate analysis (PCoA) plots were conducted on binary Jaccard and Bray Curtis 242 distance metrics, and PERMANOVA was performed to check differences between groups and $p$ 243 values were FDR adjusted after pairwise comparison. ANCOM (Mandal et al. 2015) was adopted to 244 detect statistical differences among groups at summarized L7 level using default settings in 245 QIIME2. For all taxa found to differ between treatments by ANCOM, Wilcoxon rank-sum test was 246 conducted for pairwise comparison using the respective relative abundances.

247 Phenotypic (including clinical and immunological) data were integrated with microbiota data by 248 Pearson's correlation analysis using Rhea (Lagkouvardos et al. 2017). Rare microbial appearances 249 at species level were removed with a cutoff of mean relative abundance $>0.1 \%$ and minimal 250 presence among $30 \%$ of samples. Zeros were regarded as NA. Centered log-ratio transformation 251 was conducted in both the microbial relative abundance and phenotypic data. The correlation matrix 252 was visualized with $\mathrm{R}$ package corrplot using the same defined threshold $(p<0.05)$ for both 253 platforms. 
Results

Piglet performance, blood hematology, blood biochemistry and systemic immunoglobulin

257 Piglets fed with $2.5 \%$ FRS had numerically, but not significantly increased body weight by the end 258 of the experiment ( 85 days of age) in comparison with those fed the basal diet. Further, increasing 259 the dose of FRS to 5\% did not positively influence the final body weight of animals (Table 2). No significant differences in average daily weight gain (ADWG), average weight gain (AWG), or feed conversion ratio (FCR) were recorded between the three feeding regimens. The completion rate for

262 piglets in the experiment (ie. not dead or removed due to need for antibiotics treatment) did not 263 differ between treatment groups. In the sub-group of piglets euthanized 3 weeks after weaning, we 264 found no statistical differences in blood hematology, blood chemistry and systemic 265 immunoglobulin parameters among treatment groups, except for the blood urea nitrogen (BUN) and 266 mean corpuscular characteristics (Appendix Fig. 1). Compared with piglets fed the basal diet, $2.5 \%$ 267 FRM reduced plasma level of BUN, but increased mean corpuscular volume and mean corpuscular hemoglobulin in blood. Piglets fed with 5\% FRM had a similar tendency, but only BUN level was significantly lowered relative to the basal diet.

High level of accordance between the short and long amplicon sequencing strategies

271 Two different sequencing strategies were applied. Illumina NextSeq-based amplicon sequencing of 272 the 16S rRNA gene V3 variable region (Illumina V3) and ONT based sequencing of V1-V8 273 variable regions (ONT V1-V8). Out of 99 unique taxonomic groups discovered with both methods,

27478 were shared between the two sequencing methods (Appendix Fig. 2A). The accordance between 275 the two sequencing strategies was further improved after abundance threshold adjustment 276 (Appendix Fig. 2B-D). The level of similarity between taxa identified with both sequencing 277 strategies reached $100 \%$ when analyzing taxonomic groups with relative abundance above 3\% 278 (Appendix Fig. 2E) and overall with very good correlation between the two methods (Appendix 279 Fig. 2F). Both methods revealed that the most dominant bacterial groups belonged to genus 280 Lactobacillus and families: Ruminococcae and Lachnospiraceae independent of treatment (Fig 1).

\section{Supplementation with FRS induced clear changes in the gut microbiota composition}

282 Data generated with both sequencing approaches revealed differences in gut microbial diversity in 283 piglets 3 weeks after weaning according to dietary treatment. FRS addition in the feed resulted in 
284 increased Shannon index but not Observed feature index values. The effect was consistent, when 285 the analysis was performed based on the non-summarized ASV-table (Fig 2A) and summarized to 286 the species level table (Fig. 2B) from Illumina data, and when summarized to the species level from 287 ONT data (Fig. 2C). Increasing the dietary inclusion of FRS from 2.5\% to 5\% of DM did not cause 288 significant changes in the alpha diversity indices between the two groups (Fig. 2A-C). Beta 289 diversity analysis based on binary Jaccard distance (qualitative) and Bray Curtis dissimilarity 290 (quantitative) showed that introduction of FRS in the feed influenced colon microbiota composition. 291 Surprisingly, the changes were more pronounced within the 2.5\% FRS supplemented group 292 compared to the 5\% FRS (Fig. 2D-F).

293 Prevotella stercorea and Faecalibacterium prausnitzii are positively correlated with colon 294 mucosa thickness but negatively correlated with systemic IgG levels

295 The relative abundance of Prevotella stercorea and Mitsuokella spp. were increased in the $2.5 \%$ 296 FRS-feeding group compared to basal diet and for most comparisons also in the 5\% FRS group as 297 well (Fig. 3A-D). The relative abundance of Prevotella stercorea (Illumina V3) was shown to be 298 positively correlated with the colon mucosa thickness and negatively correlated to the blood 299 leucocytes counts and serum IgG level (Fig. 4A), while this observation was only near-significant 300 using ONT (Fig. 4B).

301 There were 83 significant correlations between bacteria relative abundance verified with Illumina 302 V3 region and indicators of systemic or intestinal immunomodulation or intestinal histopathological 303 parameters, while ONT resulted in 114 significant correlations. Although many matching results 304 (associations and trends) could be found comparing correlation results from both methods, only one 305 taxon, Faecalibacterium prausnitzii presented 100\% accordance in correlations from the two 306 sequencing strategies. F. prausnitzii relative abundance correlated positively with colon mucosa 307 thickness and negatively with serum levels of aspartate aminotransferase, lactate dehydrogenase and 308 IgG (Appendix Fig. 3).

\section{Supplementation with FRS protected the gut health and alleviated inflammation}

310 The morphological characteristics of intestinal tissues obtained from piglets in the different 311 treatment groups are shown in Fig. 5A. All animals fed 2.5\%, 5\% FRS or the basal diet presented 312 normal ranges for heights and structures of villi and intestinal crypts. The continuity and height of 313 the jejunal and colonic epithelium was more pronounced in both FRS groups compared to the 314 piglets on the basal feed. Histological evaluation of tissues was performed for lymphocytic 
315 infiltration, GALT structures and colon mucosa thickness. In the jejunal epithelium and stroma, the

316 piglets fed with FRS had reduced intraepithelial lymphocytes (IEL) and stromal lymphocytes (SL)

317 infiltration compared to piglets fed the basal diet, with 2.5\% FRS showing the best effect (Fig. 5B).

318 We observed similar tendency of alleviated focal inflammation in the colon tissues of $2.5 \%$ FRS,

319 but no significant difference was found between treatment groups (Fig. 5C). Diffuse lymphoid

320 follicles at the base of the mucosa were visible with normal size and structure in jejunum and colon.

321 No clear stimulation of lymphoid follicles was observed in any of the three dietary groups. Neither

322 did FRS supplementation result in differences in the number of jejunal and colonic GALT structures

323 relative to the basal diet (Fig. 5D). The histological evaluation did not indicate damage of the

324 intestinal epithelial barrier in any group but the mucous membrane was higher with significantly

325 deeper intestinal crypts within the $2.5 \%$ FRS group compared to the basal diet group (Fig. 5E). 


\section{Discussion}

328 In modern pig production, the weaning of piglets is usually conducted at an early age causing significant stress due to changes in diet, environment and social groups. Hence, many weaned piglets experience intestinal and immune dysfunction, elevated risk of infection with enteric pathogens and hence diarrhea, and lowered weight gain due to reduced feed intake and poorer utilization of ingested nutrients (Lallès et al. 2007). Reduced weight gain and higher mortality rate among weaned piglets are highly undesirable from a production efficiency point of view. Preventive measures, such as use of in-feed antibiotics have been banned by the European Union (EU) back in 2006, while the commonly used zinc oxide $(\mathrm{ZnO})$ will be banned in 2022 . Therefore, there is an urgent need for development of alternative preventive strategies in order to sustain performance and a healthy gut of weaned piglets. We have previously reported that FRS fed to weaned piglets improved jejunal villus development, stimulated colon mucosal development and reduced signs of intestinal inflammation (Satessa et al. 2020a). Since FRS was demonstrated to be effective without in-feed $\mathrm{ZnO}$, we further investigated in the present study the dose-dependent influence of FRS on gut microbial composition and its plausible link with indicators of intestinal and systemic immune 342 function.

343 To study the gut microbial composition, we have used and compared the performance of two 344 sequencing strategies. The widely used short-read 16S rRNA gene amplicon sequencing performed on an Illumina-platform was compared with the less commonly used near-full length 16S rRNA gene sequencing method by ONT. The two strategies showed satisfying accordance and allowed to draw the same overall conclusions, including those identified at the species level, which is challenging even when different hypervariable regions of 16S rRNA gene within the same sequencing method are compared (Bukin et al. 2019) (Yang et al. 2016) (Kerrigan et al. 2019). Data generated with both sequencing strategies confirmed significant changes in gut microbiota qualitative and quantitative characteristics in response to dietary FRS supplementation. The effect was more pronounced for the $2.5 \%$ FRS group compared to the 5\% FRS group. Piglets fed the $2.5 \%$ and 5\% FRS supplemented diets had higher Shannon index indicating a more diverse and uniformly

354 distributed gut microbiome than the piglets fed basal diet. The Observed features index was largely 355 similar between all 3 groups. High microbial diversity is generally desirable, as it has been 356 demonstrated to exclude pathogenic microbes, improve immune response and reduce necrotizing 357 enterocolitis and post-weaning diarrhea incidences (Fouhse et al. 2016) (Khanna et al. 2016) (Dou 358 et al. 2017). FRS supplementation led to increased relative abundance of Prevotella stercorea and 
359 Mitsuokella spp. relative to piglets fed the basal diet, an effect that was especially pronounced in the $3602.5 \%$ FRS group. Prevotella is known to be the major contributor to the microbiome of post361 weaned piglets due to ability to degrade plant fibers in the solid diet. The species $P$. stercorea has 362 previously been described as a member of the healthy pig's GM (Wang et al. 2019) as well as a 363 potent producer of short-chain fatty acids (SCFA), when carbohydrates such as fibers are fermented 364 in the hind-gut (De Filippo et al. 2010)' (Chen et al. 2017b). Our data indicated that the abundance 365 of $P$. stercorea correlated positively with colon mucosa thickness, which is not surprising, since 366 Prevotella spp. are recognized colonizers of mucosal sites (Larsen 2017) and the produced SCFAs 367 help maintain intestinal barrier function through providing energy resources and immunoregulatory 368 regulation as well (Kiefer et al. 2006)' (Chen et al. 2017a)' (Spiljar et al. 2017). The negative 369 correlation of $P$. stercorea with the serum levels of leucocytes and $\mathrm{IgG}$ could indicate that increased 370 abundance of $P$. stercorea on the more fibrous FRS supplemented diet stimulated gut barrier and 371 immune function, and consequently relieved the host inflammation. Previous studies also report that 372 complex hemicelluloses and cellulose most likely enhances mucosal abundance of P. stercorea 373 (Mann et al. 2014) (Mach et al. 2015).

374 Faecalibacterium prausnitzii is one of the main butyrate producers found in the gastro-intestinal 375 tract (Barcenilla et al. 2000). Butyrate plays a vital role in gut physiology and gut health. It serves 376 as a main energy source for the colonocytes and plays a protective role against inflammatory 377 disease and colorectal cancer (Christl et al. 1996)' (Archer et al. 1998). Many studies have linked 378 reduced abundance of $F$. prausnitzii with different intestinal disorders, hence it has been proposed 379 that $F$. prausnitzii may serve as a biomarker of gut health (Lopez-Siles et al. 2017). Our data 380 showed that $F$. prausnitzii correlated positively with colon mucosa thickness and negatively with 381 serum levels of two enzymes released from the liver e.g. aspartate aminotransferase (AST) and 382 lactate dehydrogenase (LDH) as well as serum IgG. Increased serum level of the hepatic enzymes is 383 a sign of liver malfunction while $\mathrm{IgG}$ is systemic indicator of inflammation status of the host. 384 Hence, our findings are in line with studies demonstrating the ability of $F$. prausnitzii to reduce 385 inflammation and improve the liver function in murine models (Munukka et al. 2017) (Fukui 2019) 386 and humans.

387 Although the data on microbiota composition suggests there was no distinct impact with regards to 388 the inclusion levels of FRS (2.5\% versus $5 \%$ ), it is important to note that significantly alleviated 389 signs of lymphocyte invasion in jejunum and enhanced colon mucosa barrier function were 390 observed solely in the group receiving $2.5 \%$ FRS. Moreover, the 5\% FRS addition tended to 
391 numerically worsen production performance (ADG, FCR, completion rate). Possibly, if higher

392 amounts of FRS are added, the piglets will also be exposed to more bioactive components from

393 either the rapeseed or seaweed, which even though they are beneficial in lower amounts, could

394 increase the physiological stress in the weaning period if added in relatively high amounts and be

395 counterproductive to FCR in production. Young animals are usually more sensitive to these anti-

396 nutritional factors, like glucosinolates (Tripathi and Mishra 2007) than older animals, and thereby

397 our results suggest that the optimal inclusion level was reached at around $2.5 \%$ FRS.

398 Conclusion

399 Our study demonstrates that dietary supplementation in postweaning piglets with $2.5 \%$ FRS led to 400 significant improvements of gut health and induced favorable changes in gut microbiota 401 composition, while further increasing the dietary addition to 5\% FRS resulted in less pronounced 402 effects. The $2.5 \%$ FRS supplementation induced significant changes in gut microbial composition 403 expressed as increased microbial diversity index and elevated abundance of Prevotella stercorea 404 and Mitsuokella spp. Although clear causality cannot be proven, we found clear correlations 405 between the abundance of Prevotella stercorea and Faecalibacterium prausnitzii and biomarkers of 406 reduced intestinal and systemic inflammation, improved liver function, and increased colon 407 mucosal thickness.

\section{Acknowledgement}

409 This work was supported by Bio-Based Industries Joint Undertaking under the European Union 410 Horizon 2020 research and innovation program under grant agreement No 720755 (Macro Cascade 411 project). Gizaw Dabessa Satessa held a PhD scholarship co-financed by a grant (file no. 5157412 00003B) from the Innovation Fund Denmark and the University of Copenhagen, Denmark. Yan Hui 413 was financed by the China Scholarship Council scholarship. 


\section{Reference}

416 Archer S., Meng S., Wu J., Johnson J., Tang R., Hodin R., Ko T.C., Warner B.W., Basson M.D. Butyrate inhibits colon carcinoma cell growth through two distinct pathways. Surgery 1998;124(2):248-53.

Barcenilla A., Pryde S.E., Martin J.C., Duncan S.H., Stewart C.S., Henderson C., Flint H.J. Phylogenetic relationships of butyrate-producing bacteria from the human gut. Appl Environ Microbiol 2000;66(4):1654-61.

Bolyen E., Rideout J.R., Dillon M.R., Bokulich N.A., Abnet C.C., Al-Ghalith G.A., Alexander H., Alm E.J., Arumugam M., Asnicar F., Bai Y., Bisanz J.E., Bittinger K., Brejnrod A., Brislawn C.J., Brown C.T., Callahan B.J., Caraballo-Rodríguez A.M., Chase J., Cope E.K., Da Silva R., Diener C., Dorrestein P.C., Douglas G.M., Durall D.M., Duvallet C., Edwardson C.F., Ernst M., Estaki M., Fouquier J., Gauglitz J.M., Gibbons S.M., Gibson D.L., Gonzalez A., Gorlick K., Guo J., Hillmann B., Holmes S., Holste H., Huttenhower C., Huttley G.A., Janssen S., Jarmusch A.K., Jiang L., Kaehler B.D., Kang K. Bin, Keefe C.R., Keim P., Kelley S.T., Knights D., Koester I., Kosciolek T., Kreps J., Langille M.G.I., Lee J., Ley R., Liu Y.-X., Loftfield E., Lozupone C., Maher M., Marotz C., Martin B.D., McDonald D., McIver L.J., Melnik A. V., Metcalf J.L., Morgan S.C., Morton J.T., Naimey A.T., Navas-Molina J.A., Nothias L.F., Orchanian S.B., Pearson T., Peoples S.L., Petras D., Preuss M.L., Pruesse E., Rasmussen L.B., Rivers A., Robeson M.S., Rosenthal P., Segata N., Shaffer M., Shiffer A., Sinha R., Song S.J., Spear J.R., Swafford A.D., Thompson L.R., Torres P.J., Trinh P., Tripathi A., Turnbaugh P.J., Ul-Hasan S., van der Hooft J.J.J., Vargas F., Vázquez-Baeza Y., using QIIME 2. Nat Biotechnol 2019;37(8):852-7.

Bukin Y.S., Galachyants Y.P., Morozov I. V., Bukin S. V., Zakharenko A.S., Zemskaya T.I. The effect of $16 \mathrm{~s}$ rRNA region choice on bacterial community metabarcoding results. Sci Data 2019;6(1):1-14.

Canibe N., Højberg O., Badsberg J.H., Jensen B.B. Effect of feeding fermented liquid feed and fermented grain on gastrointestinal ecology and growth performance in piglets. J Anim Sci 2007;85(11):2959-71. 
function in a Caco-2 cell model. Food Funct 2017a;8(3):1166-73.

447

448

449

450

451

452

453

454

455

456

457

458

459

460

461

462

463

464

465

466

467

468

469

470

471

472

473

474

Chen T., Long W., Zhang C., Liu S., Zhao L., Hamaker B.R. Fiber-utilizing capacity varies in Prevotella- versus Bacteroides-dominated gut microbiota. Sci Rep 2017b;7(1):2594.

Cherry P., O’Hara C., Magee P.J., McSorley E.M., Allsopp P.J. Risks and benefits of consuming edible seaweeds. Nutr Rev 2019;77(5):307-29.

Christl S.U., Eisner H.D., Dusel G., Kasper H., Scheppach W. Antagonistic Effects of Sulfide and Butyrate on Proliferation of Colonic Mucosa: A Potential Role for These Agents in the Pathogenesis of Ulcerative Colitis. Dig Dis Sci 1996;41(12):2477-81.

DeSantis T.Z., Hugenholtz P., Larsen N., Rojas M., Brodie E.L., Keller K., Huber T., Dalevi D., Hu P., Andersen G.L. Greengenes, a chimera-checked 16S rRNA gene database and workbench compatible with ARB. Appl Environ Microbiol 2006;72(7):5069-72.

Dou S., Gadonna-Widehem P., Rome V., Hamoudi D., Rhazi L., Lakhal L., Larcher T., Bahi-Jaber N., Pinon-Quintana A., Guyonvarch A., Huërou-Luron I.L.E., Abdennebi-Najar L. Characterisation of Early-Life Fecal Microbiota in Susceptible and Healthy Pigs to PostWeaning Diarrhoea. PLoS One 2017;12(1):e0169851.

De Filippo C., Cavalieri D., Di Paola M., Ramazzotti M., Poullet J.B., Massart S., Collini S., Pieraccini G., Lionetti P. Impact of diet in shaping gut microbiota revealed by a comparative study in children from Europe and rural Africa. Proc Natl Acad Sci U S A 2010;107(33):14691-6.

Fouhse J.M., Zijlstra R.T., Willing B.P. The role of gut microbiota in the health and disease of pigs. Anim Front 2016;6(3):30-6.

Fukui H. Role of Gut Dysbiosis in Liver Diseases: What Have We Learned So Far? Diseases 2019;7(4):58.

Heres L., Engel B., Van Knapen F., De Jong M.C.M., Wagenaar J.A., Urlings H.A.P. Fermented liquid feed reduces susceptibility of broilers for Salmonella enteritidis. Poult Sci 2003;82(4):603-11.

Hu J., Lu W., Wang C., Zhu R., Qiao J. Characteristics of Solid-state Fermented Feed and its Effects on Performance and Nutrient Digestibility in Growing-finishing Pigs. AsianAustralasian J Anim Sci 2008;21(11):1635-41. 
475

476

477

478

479

480

481

482

483

484

485

486

487

488

489

490

491

492

493

494

495

496

497

498

500

501

502

503

Hu Y., Wang Y., Li A., Wang Z., Zhang X., Yun T., Qiu L., Yin Y. Effects of fermented rapeseed meal on antioxidant functions, serum biochemical parameters and intestinal morphology in broilers. Food Agric Immunol 2016;27(2):182-93.

Jha R., Berrocoso J.F.D. Dietary fiber and protein fermentation in the intestine of swine and their interactive effects on gut health and on the environment: A review. Anim Feed Sci Technol 2016;212:18-26.

Kerrigan Z., Kirkpatrick J.B., D’Hondt S. Influence of 16S rRNA Hypervariable Region on Estimates of Bacterial Diversity and Community Composition in Seawater and Marine Sediment. Front Microbiol 2019;10(JULY):1640.

Khanna S., Pardi D.S., Kelly C.R., Kraft C.S., Dhere T., Henn M.R., Lombardo M.J., Vulic M., Ohsumi T., Winkler J., Pindar C., McGovern B.H., Pomerantz R.J., Aunins J.G., Cook D.N., Hohmann E.L. A Novel Microbiome Therapeutic Increases Gut Microbial Diversity and Prevents Recurrent Clostridium difficile Infection. J Infect Dis 2016;214(2):173-81.

Kiefer J., Beyer-Sehlmeyer G., Pool-Zobel B.L. Mixtures of SCFA, composed according to physiologically available concentrations in the gut lumen, modulate histone acetylation in human HT29 colon cancer cells. Br J Nutr 2006;96(5):803-10.

Krych Ł., Kot W., Bendtsen K.M.B., Hansen A.K., Vogensen F.K., Nielsen D.S. Have you tried spermine? A rapid and cost-effective method to eliminate dextran sodium sulfate inhibition of PCR and RT-PCR. J Microbiol Methods 2018;144:1-7.

Lagkouvardos I., Fischer S., Kumar N., Clavel T. Rhea: a transparent and modular R pipeline for microbial profiling based on 16S rRNA gene amplicons. PeerJ 2017;5:e2836.

Lallès J.-P., Bosi P., Smidt H., Stokes C.R. Nutritional management of gut health in pigs around weaning. Proc Nutr Soc 2007;66(2):260-8.

Larsen J.M. The immune response to Prevotella bacteria in chronic inflammatory disease. Immunology 2017;151(4):363-74.

Lopez-Siles M., Duncan S.H., Garcia-Gil L.J., Martinez-Medina M. Faecalibacterium prausnitzii: from microbiology to diagnostics and prognostics. ISME J 2017;11(4):841-52.

Mach N., Berri M., Estellé J., Levenez F., Lemonnier G., Denis C., Leplat J.-J., Chevaleyre C., Billon Y., Doré J., Rogel-Gaillard C., Lepage P. Early-life establishment of the swine gut 
504

microbiome and impact on host phenotypes. Environ Microbiol Rep 2015;7(3):554-69.

Mandal S., Van Treuren W., White R.A., Eggesbø M., Knight R., Peddada S.D. Analysis of composition of microbiomes: a novel method for studying microbial composition. Microb Ecol Health Dis 2015;26(1):27663.

Mann E., Schmitz-Esser S., Zebeli Q., Wagner M., Ritzmann M., Metzler-Zebeli B.U. Mucosaassociated bacterial microbiome of the gastrointestinal tract of weaned pigs and dynamics linked to dietary calcium-phosphorus. PLoS One 2014;9(1):e86950.

Mukherjee R., Chakraborty R., Dutta A. Role of Fermentation in Improving Nutritional Quality of Soybean Meal - A Review. Asian-Australasian J Anim Sci 2015;29(11):1523-9.

Munukka E., Rintala A., Toivonen R., Nylund M., Yang B., Takanen A., Hänninen A., Vuopio J., Huovinen P., Jalkanen S., Pekkala S. Faecalibacterium prausnitzii treatment improves hepatic health and reduces adipose tissue inflammation in high-fat fed mice. ISME J 2017;11(7):166779.

Peterson L.W., Artis D. Intestinal epithelial cells: regulators of barrier function and immune homeostasis. Nat Rev Immunol 2014;14(3):141-53.

Plumed-Ferrer C., von Wright A. Fermented pig liquid feed: nutritional, safety and regulatory aspects. J Appl Microbiol 2009;106(2):351-68.

Satessa G.D., Tamez-Hidalgo P., Hui Y., Cieplak T., Krych L., Kjærulff S., Brunsgaard G., Nielsen D.S., Nielsen M.O. Impact of Dietary Supplementation of Lactic Acid Bacteria Fermented Rapeseed with or without Macroalgae on Performance and Health of Piglets Following Omission of Medicinal Zinc from Weaner Diets. Animals 2020a;10(1):137.

Satessa G.D., Tamez-Hidalgo P., Kjærulff S., Vargas-Bello-Pérez E., Dhakal R., Nielsen M.O. Effects of Increasing Doses of Lactobacillus Pre-Fermented Rapeseed Product with or without Inclusion of Macroalgae Product on Weaner Piglet Performance and Intestinal Development. Animals 2020b;10(4):559.

Scudellari M. News Feature: Cleaning up the hygiene hypothesis. Proc Natl Acad Sci 2017;114(7):1433-6.

van der Spiegel M., Noordam M.Y., van der Fels-Klerx H.J. Safety of Novel Protein Sources (Insects, Microalgae, Seaweed, Duckweed, and Rapeseed) and Legislative Aspects for Their 
Application in Food and Feed Production. Compr Rev Food Sci Food Saf 2013;12(6):662-78.

534

535

536

537

538

539

540

541

542

543

544

545

546

547

548

549

550

551

552

553

554

555

556

557

Spiljar M., Merkler D., Trajkovski M. The Immune System Bridges the Gut Microbiota with Systemic Energy Homeostasis: Focus on TLRs, Mucosal Barrier, and SCFAs. Front Immunol 2017;8(OCT):1353.

Tripathi M.K., Mishra A.S. Glucosinolates in animal nutrition: A review. Anim Feed Sci Technol 2007;132(1-2):1-27.

Tybirk P.E.R. Nutrient recommendations for pigs in Denmark. Danis Pig Res Cent 2015;

Wang C., Shi C., Zhang Y., Song D., Lu Z., Wang Y. Microbiota in fermented feed and swine gut. Appl Microbiol Biotechnol 2018;102(7):2941-8.

Wang J., Han Y., Zhao J.Z., Zhou Z.J., Fan H. Pyrosequencing-based analysis of the complex microbiota located in the gastrointestinal tracts of growing-finishing pigs. Anim Prod Sci 2019;59(5):870.

Xu B., Li Z., Wang C., Fu J., Zhang Y., Wang Y., Lu Z. Effects of fermented feed supplementation on pig growth performance: A meta-analysis. Anim Feed Sci Technol 2020;259:114315.

Yang B., Wang Y., Qian P.Y. Sensitivity and correlation of hypervariable regions in 16S rRNA genes in phylogenetic analysis. BMC Bioinformatics 2016;17(1):135.

Zargarzadeh M., Amaral A.J.R., Custódio C.A., Mano J.F. Biomedical applications of laminarin. Carbohydr Polym 2020;232:115774.

Zeng H. Mechanisms linking dietary fiber, gut microbiota and colon cancer prevention. World J Gastrointest Oncol 2014;6(2):41.

Zhou H., Wang C., Ye J., Chen H., Tao R. Effects of dietary supplementation of fermented Ginkgo bilobaL. residues on growth performance, nutrient digestibility, serum biochemical parameters and immune function in weaned piglets. Anim Sci J 2015;86(8):790-9. 


\section{$558 \quad$ Figures}

559 Fig. 1. Relative abundance of prokaryotes based on 16S rRNA gene amplicon sequencing

560

561

562

563

564

565

566

567

568

569

570

571

572

573

574

575

576

577

578

579

580

581

582

583

584

585

586

587

588 using either the Illumina or the Oxford Nanopore Technologies platform. Respectively $\mathrm{n}=9,8$, 10 for basal diet with $0 \%, 2.5 \%$ and $5 \%$ added FRS (fermented rapeseed- Sacharina latissimaAscophillum nodossum).

Fig. 2. Alpha and beta diversity analysis of $16 \mathrm{~S}$ rRNA gene amplicon sequencing data. Observed ASVs and Shannon index based on rarefied ASV table with Illumina sequencing on V3 region (Illumina V3) (A); Observed features and Shannon index based on species-level summarized table with Illumina sequencing on V3 region (B) and Oxford Nanopore sequencing on V1-V8 region (ONT V1-V8) (C). The mean value for each group is marked as a bold line respectively. Respective PCoA plot of binary Jaccard and Bray Curtis distance metrics based on rarefied ASV table (Illumina V3) (D), species-level summarized table (Illumina V3) (E) and species-level summarized table (ONT V1-V8) (F). The ellipses show respective 80\% confidential area following multivariate t-distribution. Respectively $\mathrm{n}=9,8,10$ for basal diet with $0 \%, 2.5 \%$ and $5 \%$ added FRS (fermented rapeseed- Sacharina latissima-Ascophillum nodossum). For pairwise Wilcoxon test on alpha diversity, the labels of $* * *$ represent $p<0.05,<0.01$ respectively. For PERMANOVA tests, $p$ values below 0.05 were heighted in yellow.

Fig. 3. Prevotella stercorea and Mitsuokella spp. showed different colonized abundance in gut between feeding regime groups by both Illumina sequencing on $\mathrm{V} 3$ region of $16 \mathrm{~S}$ rRNA gene $(A, B)$ and Oxford Nanopore sequencing on V1-V8 region of 16S rRNA gene $(C, D)$. Data in the bar plot was presented as mean value and SEM error bar. Respectively $n=9,8,10$ for basal diet with $0 \%, 2.5 \%$ and 5\% added FRS (fermented rapeseed- Sacharina latissima- Ascophillum nodossum). The labels of $* * *, * * *$ represent $p<0.05,<0.01,<0.005$ respectively.

Fig. 4. Prevotella stercorea significantly correlated with colon mucosa thickness, serum leucocyte and IgG with data from $\mathrm{V} 3$ region $16 \mathrm{~S}$ rRNA gene sequencing $(\mathrm{A})$, similar trend yet not significant was observed using V1-V8 region $16 \mathrm{~S}$ rRNA gene sequencing (B). Data was centered log-ratio transformed for Pearson's correlation.

Fig. 5. 2.5\% FRS feeding protected the gut health of piglets with alleviated inflammation symptoms and enhanced mucosa barrier in gut. Histopathological micrograph of jejunum and colon under 10x magnification (A), levels of inflammation measured by the number of lymphocytes infiltrated at the epithelium (IEL) and at the stroma (SL) of jejunum (B) and colon (C), levels of 
589 immune capacity estimated by numbers of lymphoid follicles per squared millimeter in jejunum and

590 colon (D) and mucosa thickness in colon (E). Respectively $n=9,8,10$ for basal diet with $0 \%, 2.5 \%$

591 and 5\% added FRS (fermented rapeseed- Sacharina latissima- Ascophillum nodossum). The labels

592 of $* * *, * * *$ represent $p<0.05,<0.01,<0.005$ respectively.

593 
594 Tables

595 Table 1. Feed formulations used for the experiment.

\begin{tabular}{|c|c|c|c|c|c|c|}
\hline & \multicolumn{3}{|c|}{ Pre-starter diet } & \multicolumn{3}{|c|}{ Starter diet } \\
\hline & $\begin{array}{l}\text { Basal } \\
\text { diet }\end{array}$ & $2.5 \% \mathrm{FRS}$ & $5 \% \mathrm{FRS}$ & $\begin{array}{l}\text { Basal } \\
\text { diet }\end{array}$ & $2.5 \% \mathrm{FRS}$ & $5 \% \mathrm{FRS}$ \\
\hline Ingredients & $(\mathrm{g} / \mathrm{kg})$ & & & & & \\
\hline Wheat $(11.2 \%)$ & 613.26 & 597.88 & 583.86 & 539.88 & 525.11 & 508.24 \\
\hline Barley $(10.6 \%)$ & 100.00 & 100.00 & 100.00 & 200.00 & 200.00 & 200.00 \\
\hline Soybean meal $(46.0 \%)$ & 0.00 & 0.00 & 0.00 & 170.00 & 170.00 & 169.69 \\
\hline Digestible soy & 79.08 & 69.35 & 59.32 & 10.63 & 0.77 & 0.00 \\
\hline $\begin{array}{l}\text { Fermented rapeseed- } \\
\text { seaweed meal }\end{array}$ & 0.00 & 25.00 & 50.00 & 0.00 & 25.00 & 50.00 \\
\hline Potato protein & 40.00 & 40.00 & 40.00 & 0.00 & 0.00 & 0.00 \\
\hline Fish meal (70\%) & 40.00 & 40.00 & 40.00 & 14.00 & 14.00 & 7.00 \\
\hline Whey protein & 50.00 & 50.00 & 50.00 & 0.00 & 0.00 & 0.00 \\
\hline Soy bean oil & 37.28 & 38.93 & 40.17 & 23.74 & 25.20 & 26.50 \\
\hline Limestone (Ca 38.5\%) & 0.00 & 0.00 & 0.00 & 5.71 & 5.57 & 5.66 \\
\hline Calcium formate & 5.00 & 5.00 & 5.00 & 0.00 & 0.00 & 0.00 \\
\hline Calcium phosphate & 9.12 & 8.68 & 8.23 & 8.83 & 8.38 & 8.29 \\
\hline Sodium chloride & 3.83 & 3.70 & 3.58 & 4.98 & 4.85 & 4.89 \\
\hline Summer fruit & 2.00 & 2.00 & 2.00 & 2.00 & 2.00 & 2.00 \\
\hline Tretracid liquid & 5.82 & 5.83 & 5.84 & 5.60 & 5.61 & 5.75 \\
\hline Lysine $\mathrm{HCl}(98 \%)$ & 5.00 & 4.00 & 2.50 & 5.00 & 4.00 & 2.50 \\
\hline Methionine DL (99\%) & 0.77 & 0.73 & 0.69 & 1.02 & 0.97 & 1.01 \\
\hline Threonine L (99\%) & 1.88 & 1.83 & 1.77 & 1.94 & 1.88 & 1.85 \\
\hline Valine $(98 \%)$ & 1.11 & 1.09 & 1.06 & 1.00 & 0.97 & 0.96 \\
\hline Tryptophan (99\%) & 0.65 & 0.65 & 0.65 & 0.37 & 0.37 & 0.36 \\
\hline Microbial phytase & 0.15 & 0.15 & 0.15 & 0.15 & 0.15 & 0.15 \\
\hline $\begin{array}{l}\text { Microbial xylanase, } \\
\text { beta-glucanase }\end{array}$ & 0.15 & 0.15 & 0.15 & 0.15 & 0.15 & 0.15 \\
\hline Vitamin E $(50 \%)$ & 0.03 & 0.03 & 0.30 & 0.10 & 0.01 & 0.01 \\
\hline Vitamin-mineral premix ${ }^{\mathrm{a}}$ & 5.00 & 5.00 & 5.00 & 5.00 & 5.00 & 5.00 \\
\hline $\begin{array}{ll}\text { Calculated nutritive } \\
\text { value }\end{array}$ & $\%$ & & & & & \\
\hline Dry weight & 88.50 & 88.50 & 88.60 & 87.50 & 87.50 & 87.60 \\
\hline $\begin{array}{l}\text { Metabolizable energy } \\
\text { (MJ) }\end{array}$ & 14.30 & 14.30 & 14.30 & 13.50 & 13.50 & 13.50 \\
\hline Crude protein & 19.70 & 19.70 & 19.70 & 18.40 & 18.40 & 18.40 \\
\hline Crude fat & 5.56 & 5.75 & 5.90 & 4.03 & 4.20 & 4.31 \\
\hline Crude fiber & 2.20 & 2.38 & 2.57 & 3.11 & 3.29 & 3.51 \\
\hline Ashes & 5.37 & 5.42 & 5.45 & 5.69 & 5.71 & 5.87 \\
\hline Starch (g) & 471.40 & 408.30 & 399.90 & 425.60 & 416.90 & 406.80 \\
\hline Lactose $(\mathrm{g})$ & 36.50 & 36.50 & 36.50 & 0.00 & 0.00 & 0.00 \\
\hline Calcium & 0.83 & 0.83 & 0.84 & 0.82 & 0.82 & 0.82 \\
\hline Total phosphorous & 0.65 & 0.65 & 0.65 & 0.58 & 0.58 & 0.58 \\
\hline Digestible phosphorous & 0.59 & 0.59 & 0.59 & 0.51 & 0.51 & 0.51 \\
\hline
\end{tabular}




\begin{tabular}{|c|c|c|c|c|c|c|}
\hline Sodium & 0.23 & 0.23 & 0.23 & 0.22 & 0.22 & 0.22 \\
\hline Chlorine & 0.57 & 0.56 & 0.56 & 0.51 & 0.50 & 0.50 \\
\hline Potassium & 0.65 & 0.65 & 0.66 & 0.67 & 0.68 & 0.69 \\
\hline Lysine & 1.46 & 1.46 & 1.46 & 1.28 & 1.28 & 1.28 \\
\hline Methionine & 0.45 & 0.45 & 0.45 & 0.41 & 0.41 & 0.41 \\
\hline Met + Cyst $^{b}$ & 0.77 & 0.77 & 0.78 & 0.72 & 0.73 & 0.73 \\
\hline Threonine & 0.92 & 0.92 & 0.92 & 0.81 & 0.81 & 0.81 \\
\hline Tryptophane & 0.30 & 0.31 & 0.31 & 0.26 & 0.26 & 0.26 \\
\hline Valine & 1.03 & 1.03 & 1.03 & 0.90 & 0.90 & 0.90 \\
\hline Isoleucine & 0.78 & 0.78 & 0.78 & 0.70 & 0.69 & 0.69 \\
\hline
\end{tabular}


598 Table 2. Performance of piglets subjected to three dietary regimens.

\begin{tabular}{lllllll}
\hline Parameters & $\begin{array}{l}\text { Basal diet } \\
(0 \% \text { added })\end{array}$ & $\begin{array}{l}2.5 \% \text { FRS } \\
(2.5 \% \text { added })\end{array}$ & $\begin{array}{l}5 \% \text { FRS } \\
(5 \% \text { added })\end{array}$ & SEM & & \multicolumn{2}{c}{ P-value } \\
\cline { 5 - 6 } & & & TG & \multicolumn{2}{c}{ TG } \\
\cline { 5 - 6 } & & & & L & Q \\
\hline
\end{tabular}

\begin{tabular}{llll}
\hline Weaning weight & $6.07 \pm 0.33$ & $6.24 \pm 0.34$ & $5.99 \pm 0.66$
\end{tabular}

$(\mathrm{kg}$, at $\mathrm{d} 28)$

\begin{tabular}{llllllll}
\multicolumn{7}{c}{ Body weight, at age of interest $(\mathrm{kg})$} \\
42 & 6.77 & 7.00 & 6.55 & 0.406 & 0.579 & 0.890 & 0.428 \\
49 & 8.24 & 8.75 & 8.41 & 0.418 & 0.563 & 0.746 & 0.314 \\
77 & 20.5 & 21.6 & 20.2 & 1.18 & 0.537 & 0.842 & 0.341 \\
85 & 23.2 & 25.1 & 23.7 & 1.82 & 0.467 & 0.823 & 0.269
\end{tabular}

\begin{tabular}{|c|c|c|c|c|c|c|c|}
\hline \multicolumn{8}{|c|}{ ADFI (kg/day) } \\
\hline 28 - 42 days & 0.162 & 0.183 & 0.166 & 0.015 & 0.437 & 0.830 & 0.231 \\
\hline $28-49$ days & 0.233 & 0.232 & 0.230 & 0.020 & 0.985 & 0.900 & 0.967 \\
\hline 28 - 85 days & 0.516 & 0.572 & 0.546 & 0.037 & 0.418 & 0.477 & 0.236 \\
\hline $50-77$ days & 0.626 & 0.661 & 0.632 & 0.031 & 0.531 & 0.879 & 0.299 \\
\hline 50 - 85 days & 0.671 & 0.763 & 0.719 & 0.058 & 0.365 & 0.460 & 0.199 \\
\hline \multicolumn{8}{|c|}{ ADG (kg/day) } \\
\hline 28 - 42 days & 0.049 & 0.063 & 0.049 & 0.025 & 0.835 & 0.993 & 0.574 \\
\hline $28-49$ days & 0.103 & 0.122 & 0.118 & 0.017 & 0.681 & 0.506 & 0.499 \\
\hline 28 - 85 days & 0.306 & 0.339 & 0.316 & 0.034 & 0.584 & 0.790 & 0.343 \\
\hline 50 - 77 days & 0.439 & 0.456 & 0.420 & 0.037 & 0.623 & 0.702 & 0.476 \\
\hline 50 - 85 days & 0.423 & 0.467 & 0.431 & 0.045 & 0.486 & 0.993 & 0.574 \\
\hline \multicolumn{8}{|c|}{ FCR } \\
\hline 28 - 42 days & 6.78 & 7.42 & 7.13 & 3.12 & 0.938 & 0.878 & 0.740 \\
\hline 28 - 49 days & 2.37 & 2.12 & 2.09 & 0.311 & 0.681 & 0.486 & 0.683 \\
\hline 28 - 85 days & 1.80 & 1.68 & 1.76 & 0.147 & 0.584 & 0.834 & 0.515 \\
\hline $50-77$ days & 1.44 & 1.45 & 1.55 & 0.084 & 0.485 & 0.365 & 0.597 \\
\hline 50 - 85 days & 6.78 & 7.42 & 7.13 & 3.12 & 0.938 & 0.878 & 0.740 \\
\hline \multicolumn{8}{|c|}{ Completion rate (\%) } \\
\hline 28 - 42 days & 95.2 & 96.9 & 96.1 & 2.10 & 0.773 & 0.74 & 0.504 \\
\hline $28-49$ days & 94.1 & 95.1 & 94.3 & 2.29 & 0.847 & 0.921 & 0.601 \\
\hline 28 - 85 days & 94.8 & 94.4 & 89.1 & 3.60 & 0.472 & 0.328 & 0.564 \\
\hline
\end{tabular}

$599 \mathrm{ADFI}=$ average daily feed intake; $\mathrm{ADG}=$ average daily gain; $\mathrm{FCR}=$ feed conversion ratio; $\mathrm{TG}=$

600 treatment group; $\mathrm{L}=$ linear effect; $\mathrm{Q}=$ quadratic effect; The significance between different dosage

601 group is based on the result of orthogonal contrasts.

602 


\section{Appendix}

604 Appendix Table 1. PCR primer sequence for V1-V8 region 16S rRNA gene sequencing with 605 Oxford Nanopore Technologies platform.

\begin{tabular}{|l|l|}
\hline Barcode-ID & sequence (5'-3') \\
\hline BC02 & GTTAGTTGATGTAGTACAGACGACTACAAACGGAATCGAGTCTCGTGGGCTCGG \\
BC06 & GTTAGTTGATGTAGTGACTACTTTCTGCCTTTGCGAGAAGTCTCGTGGGCTCGG \\
BC14 & TACATTGATGCATGGTCTATGGGTCCCAAGAGACTCGTTGTCTCGTGGGCTCGG \\
BC16 & TACATTGATGCATGGAGTACGAACCACTGTCAGTTGACGGTCTCGTGGGCTCGG \\
BC17 & GTCTCGTCCGCTCGGATCAGAGGTACTTTCCTGGAGGGTGTCTCGTGGGCTCGG \\
BC19 & GTTAGTTGATGTAGTATCTCTTGACACTGCACGAGGAACGTCTCGTGGGCTCGG \\
BC28 & GTTAGTTGATGTAGTGATACGGTGCCTTCTTAGGTTTCAGTCTCGTGGGCTCGG \\
BC30 & GTTAGTTGATGTAGTTGGGTCGAAGTAGATCCTCACTGAGTCTCGTGGGCTCGG \\
BC32 & GTTAGTTGATGTAGTATGACGTTGTCGGACTTCTACTGGGTCTCGTGGGCTCGG \\
BC37 & GTCTCGTCCGCTCGGTGATACTAAGCATCAATCGCAAGCGTCTCGTGGGCTCGG \\
BC38 & GTTAGTTGATGTAGTTTCTCTGTATCGTCCTCCTGTGGTGTCTCGTGGGCTCGG \\
BC42 & GTCTCGTCCGCTCGGCTGGCAGGTATGCCTTACACGTAGGTCTCGTGGGCTCGG \\
BC44 & GTTAGTTGATGTAGTGAGTGGGAAGGAACCCTTTCTACTGTCTCGTGGGCTCGG \\
BC52 & TACATTGATGCATGGGTCCACGAACAATCTTGTCTCTCAGTCTCGTGGGCTCGG \\
BC55 & GTCTCGTCCGCTCGGCGTAGATCAGGGTCTCATCTTCCAGTCTCGTGGGCTCGG \\
BC56 & GTCTCGTCCGCTCGGTTCATGCCACCTGTTGAGTAGTGAGTCTCGTGGGCTCGG \\
BC57 & TACATTGATGCATGGACTTCCGAAGGAGATTGACCTAGCGTCTCGTGGGCTCGG \\
BC64 & GTTAGTTGATGTAGTGAAAGTCGATGAACGGTGTCTGTCGTCTCGTGGGCTCGG \\
BC65 & GTCTCGTCCGCTCGGCCTTGTCTGGAGGAAGACTGAGAAGTCTCGTGGGCTCGG \\
BC67 & TACATTGATGCATGGGGTGAGCACACGAGTATGACAAACGTCTCGTGGGCTCGG \\
BC70 & GTTAGTTGATGTAGTACACTCCATTCGTAGGATCTCGGTGTCTCGTGGGCTCGG \\
BC72 & GTTAGTTGATGTAGTGTCGGTATGGAAGACAGTCAGCTAGTCTCGTGGGCTCGG \\
BC74 & GTTAGTTGATGTAGTAGTGGAAGTGTTGGGATGCTTGTAGTCTCGTGGGCTCGG \\
BC76 & GTTAGTTGATGTAGTGTCCAGGGTTGATGTAACAAGCATGTCTCGTGGGCTCGG \\
BC77 & GTCTCGTCCGCTCGGGTTGTATCCCTGAGAAACAGGTCGGTCTCGTGGGCTCGG \\
BC78 & GTTAGTTGATGTAGTTTCTGATTCAAAGGTTCGGTTGTTGTCTCGTGGGCTCGG \\
BC79 & GTCTTCCGCTCGGCAGCAGTGAGAACTATCTCCGAGAGTCTCGTGGGCTCGG \\
\hline
\end{tabular}

606 Appendix Fig. 1 Hierarchical clustered heatmap showing the hematological data of piglets

607 under experimental diets: basal diet, 2.5\% and 5\% FRS. Respectively $n=10,10,10$ for basal 608 diet with 0\%, 2.5\% and 5\% added FRS (fermented rapeseed- Sacharina latissima-Ascophillum 609 nodossum). The $p$ values from pairwise comparison is showed by color depth and the labels of $*$ 610 represent $p<0.05$. 
611 Appendix Fig. 2. Shared species between the short-read and long-read amplicon sequencing

612 (A-E) and upper triangle heatmap of Pearson's correlation within the shared taxa (F). Venn

613 plots of species-level summarized features from Illumina and ONT sequencing with mean relative

614 abundance cut-off of $0 \%$ (A), $0.1 \%$ (B), 1\% (C), 2\% (D), 3\% (E). The annotations of Illumina

615 species on rarefied ASVs, Illumina rarefied species and ONT indicate the captured labels at lowest

616 taxonomic level using the rarefied Illumina ASV table, Illumina and ONT species-level

617 summarized tables respectively.

618 Appendix Fig. 3. Heatmap showing Pearson's correlation between the hematological 619 parameters and bacteria relative abundance calculated from $\mathrm{V} 3$ region sequencing of $16 \mathrm{~S}$ 620 rRNA gene and V1-V8 region sequencing of 16S rRNA gene. The color depth and size of the 621 points represent the coefficient and $p$ value respectively. Statistically significant pairs with $p<0.05$

622 are marked with *. 


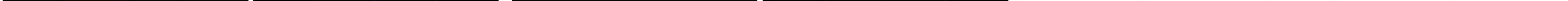




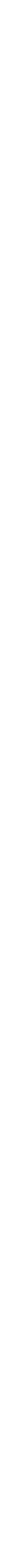


A Detected biological sequence summarized at species level

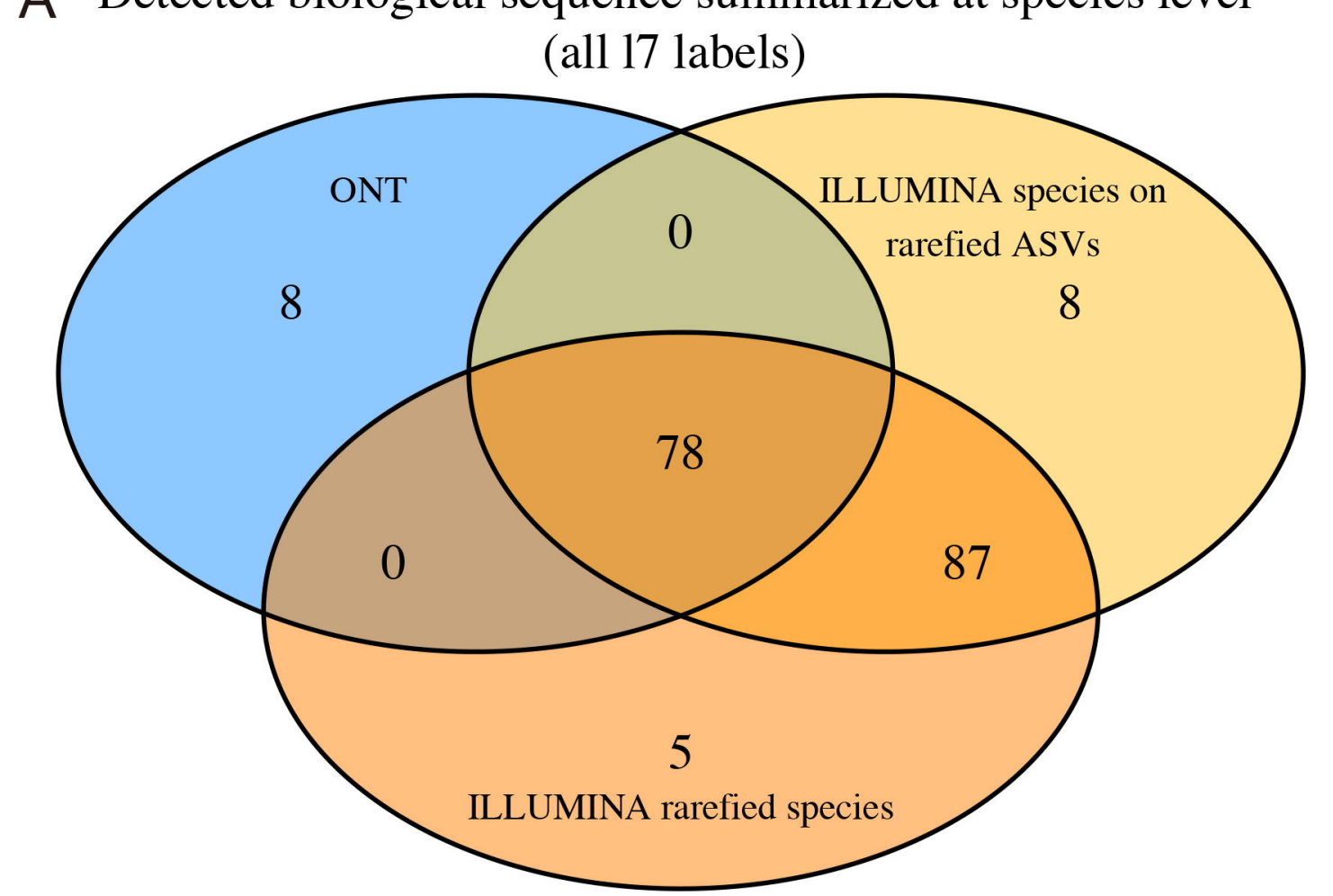

B Detected biological sequence summarized at species level (17 labels with mean relative abundance $>0.1 \%$ )

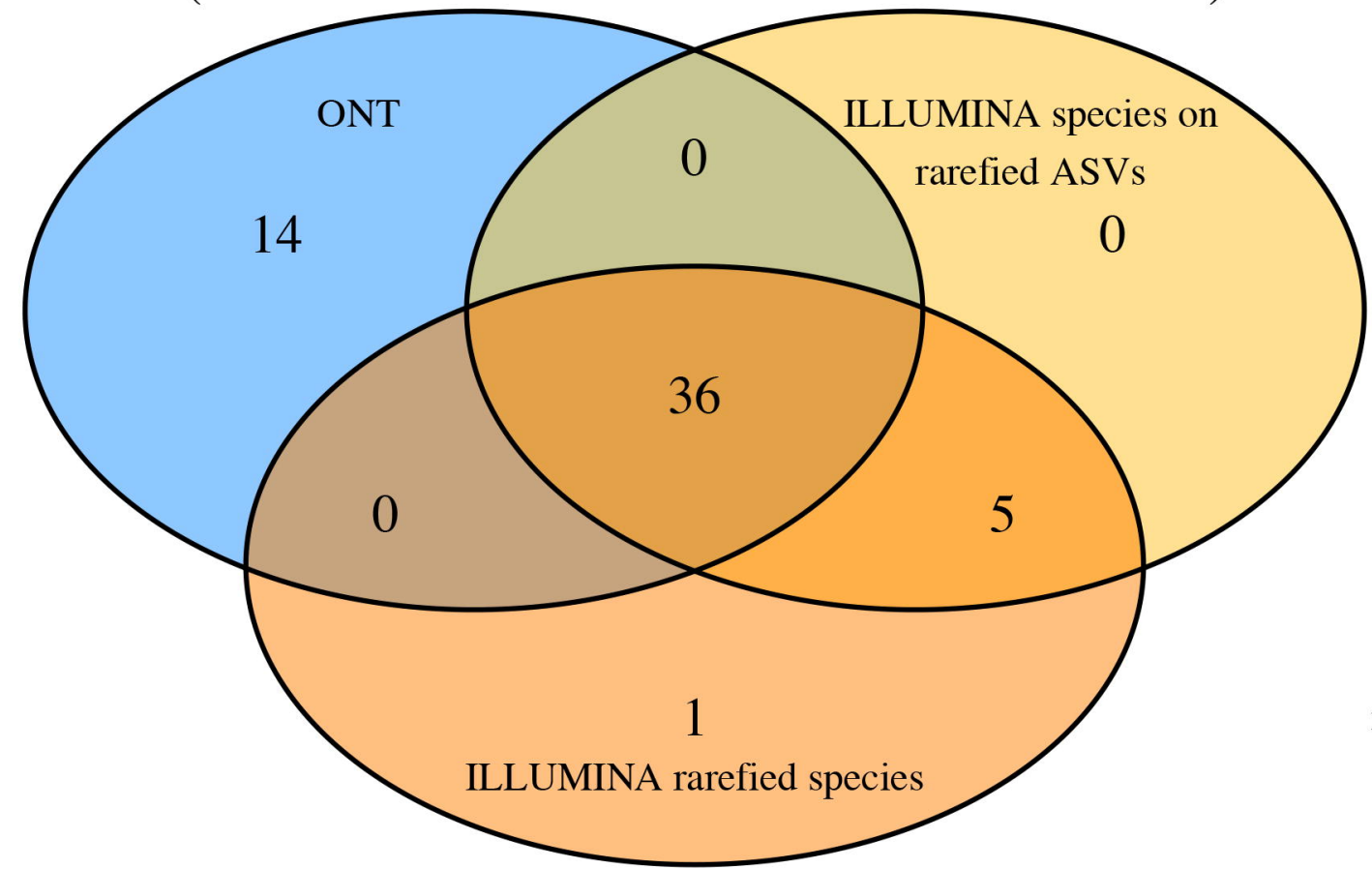

C Detected biological sequence summarized at species levels with mean relative abundance $>1 \%)$
(17 labels

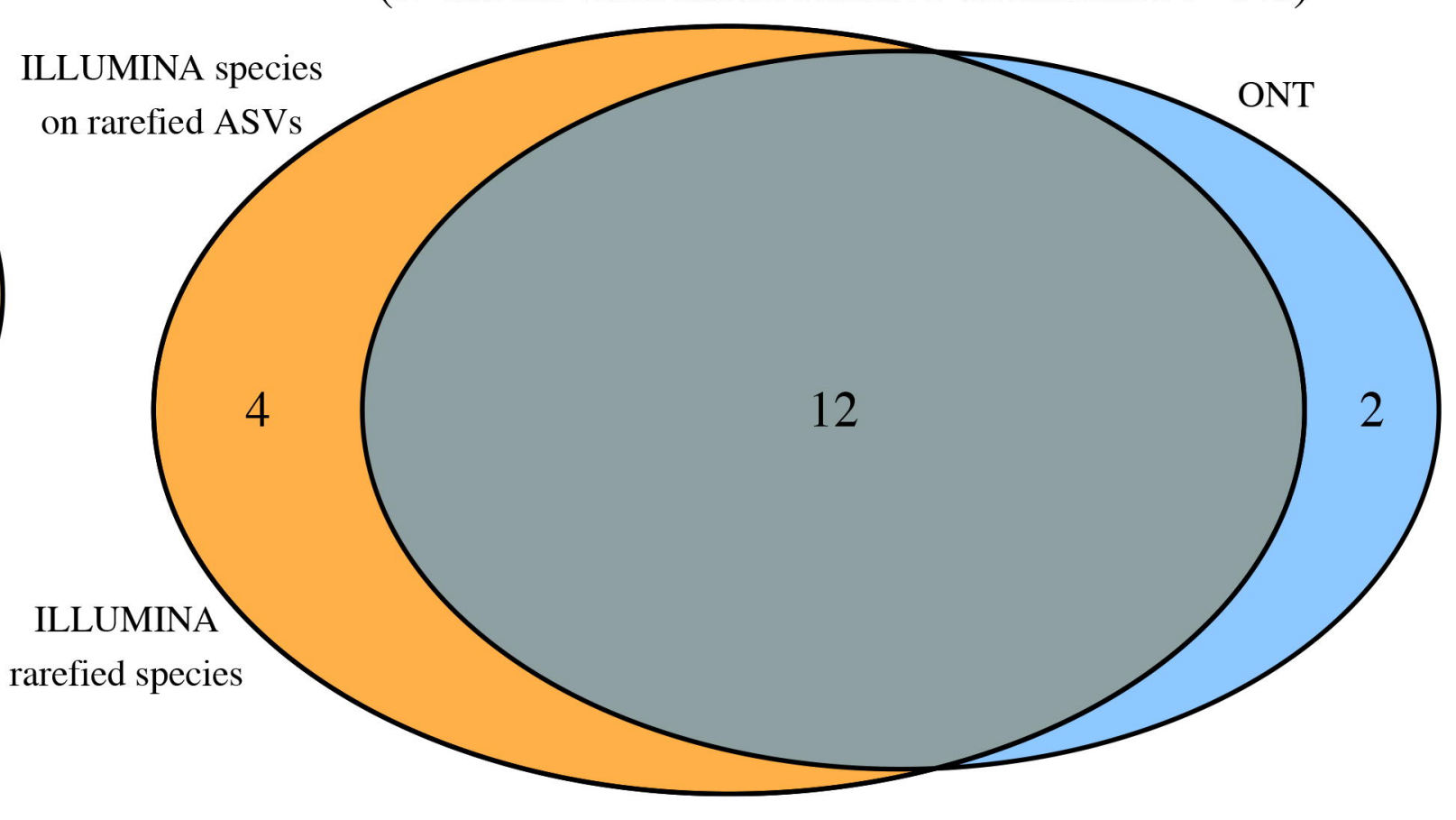

D Detected biological sequence summarized at species level (17 labels with mean relative abundance $>2 \%$ )

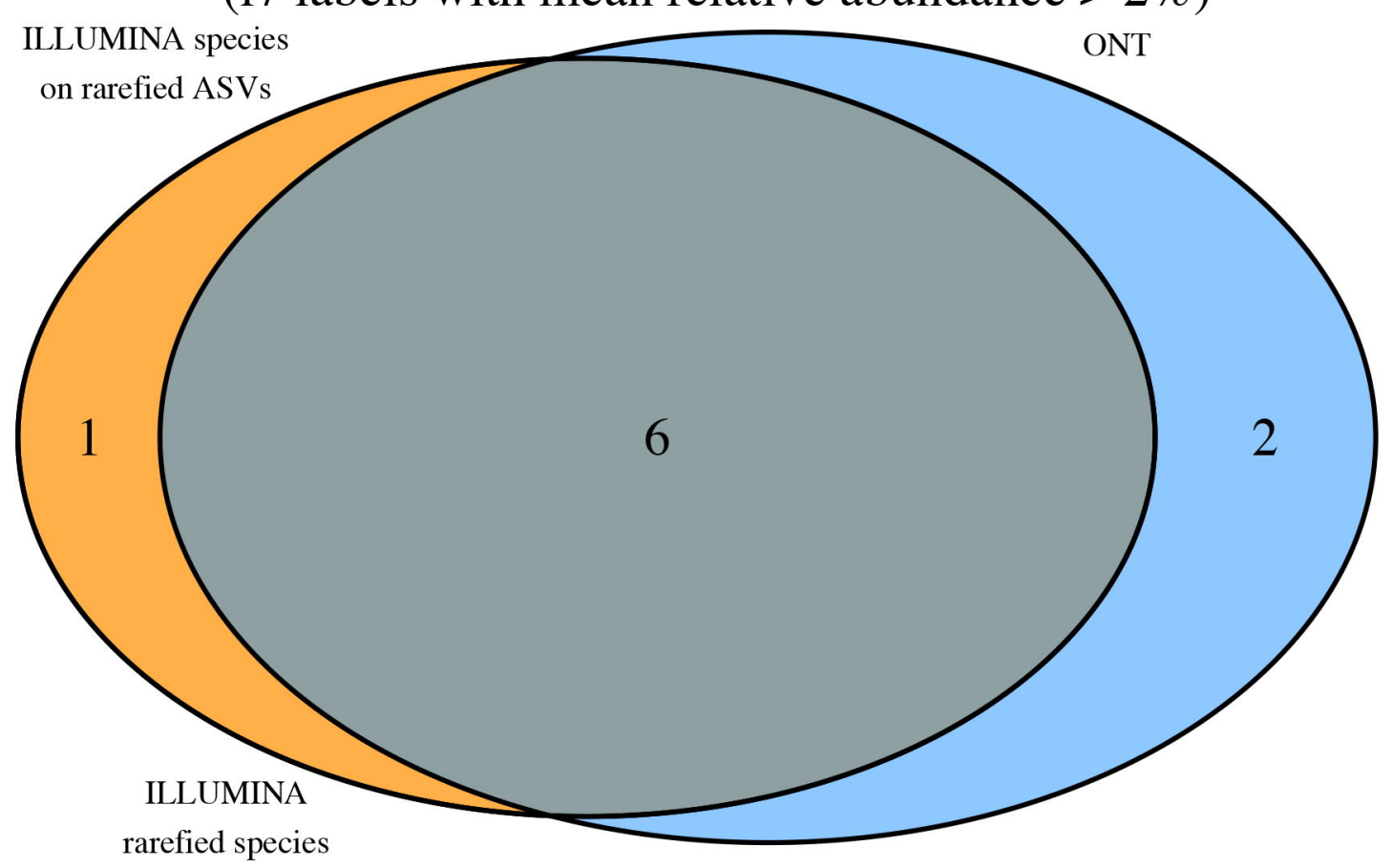

E Detected biological sequence summarized at species level (17 labels with mean relative abundance $>3 \%$ )

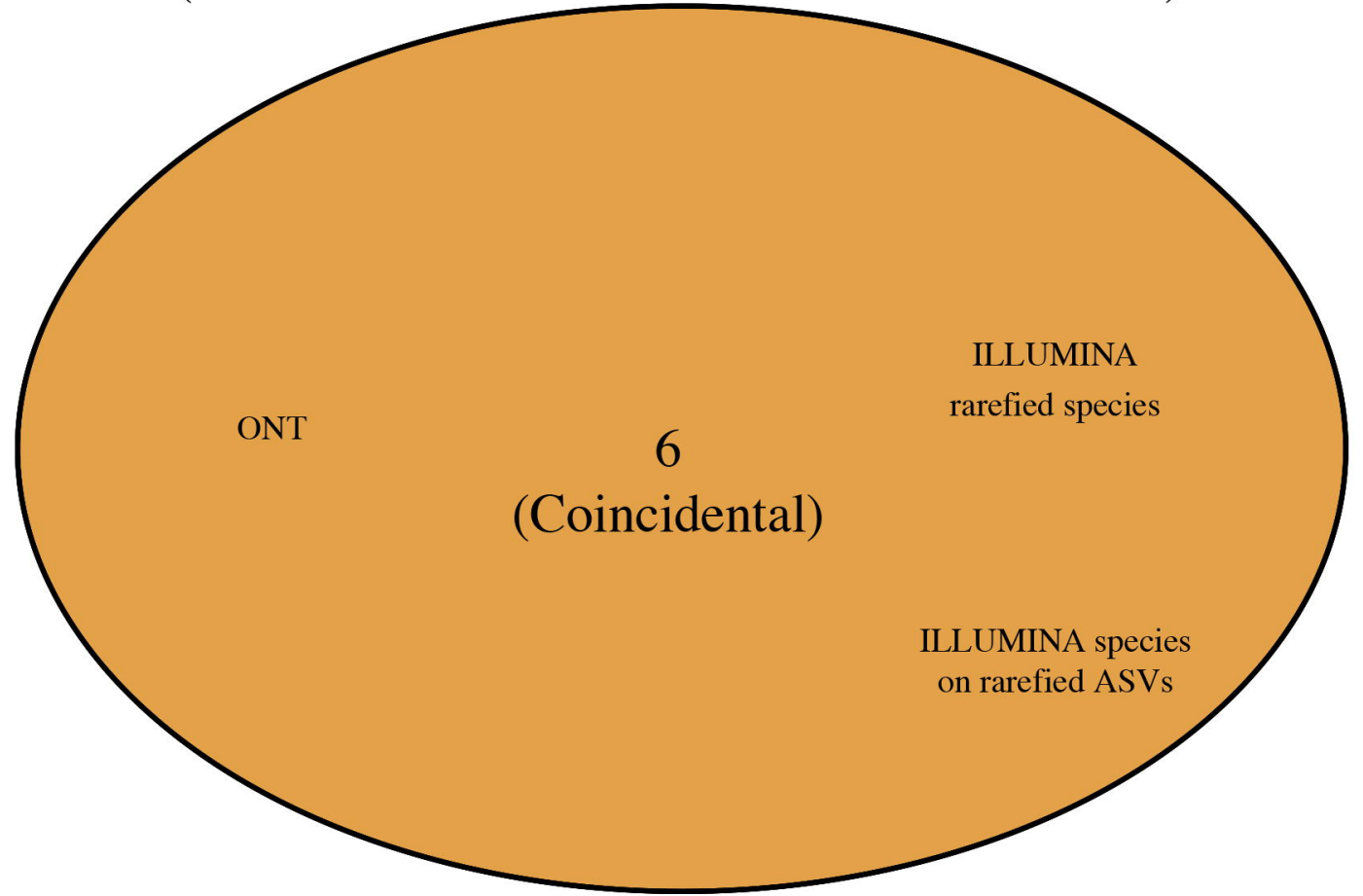

$\mathrm{F}$

S_unknown_Clostridiaceae(ILLUMINA)

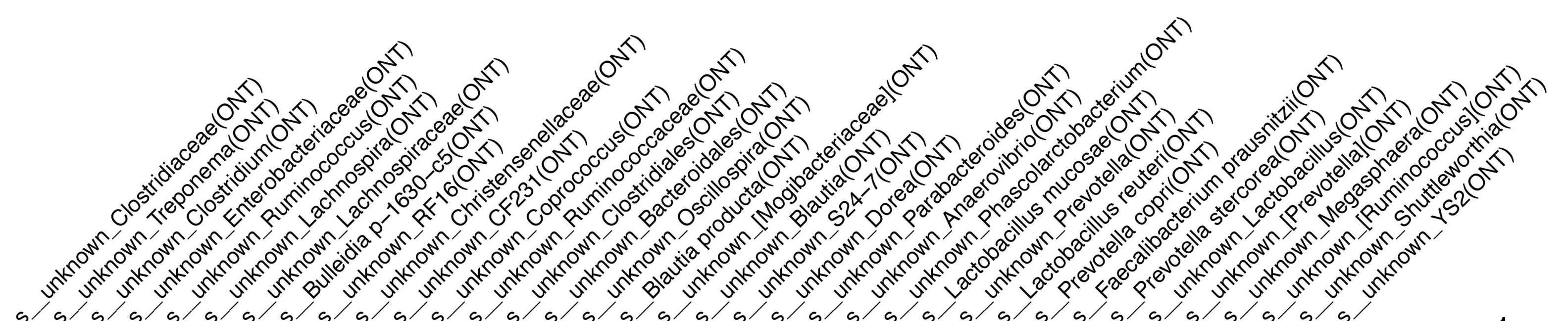

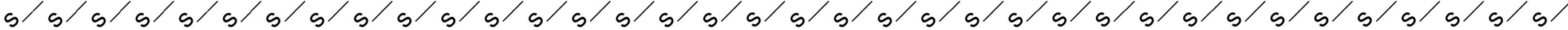

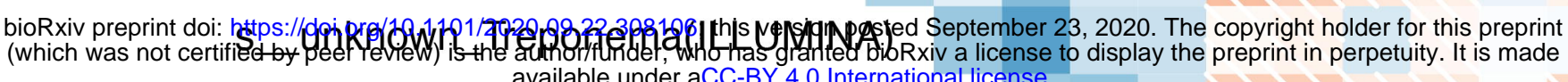

S_unknown_Clostridium(ILLCUMINA)

s_unknown_Enterobacteriaceae(ILLUMINA)

S_unknown_Ruminococcus(ILLUMINA)

s_unknown_Lachnospira(ILLUMINA)

S_unknown_Lachnospiraceae(ILLUMINA)

s_Bulleidia p-1630-c5(ILLUMINA)

S_unknown_RF16(ILLUMINA)

s_unknown_Christensenellaceae(ILLUMINA)

S_unknown_CF231(ILLUMINA)

s_unknown_Coprococcus(ILLUMINA)

s_unknown_Ruminococcaceae(ILLUMINA)

s_unknown_Clostridiales(ILLUMINA)

s_unknown_Bacteroidales(ILLUMINA)

S_unknown_Oscillospira(ILLUMINA)

S_Blautia producta(ILLUMINA)

s_unknown_[Mogibacteriaceae](ILLUMINA)

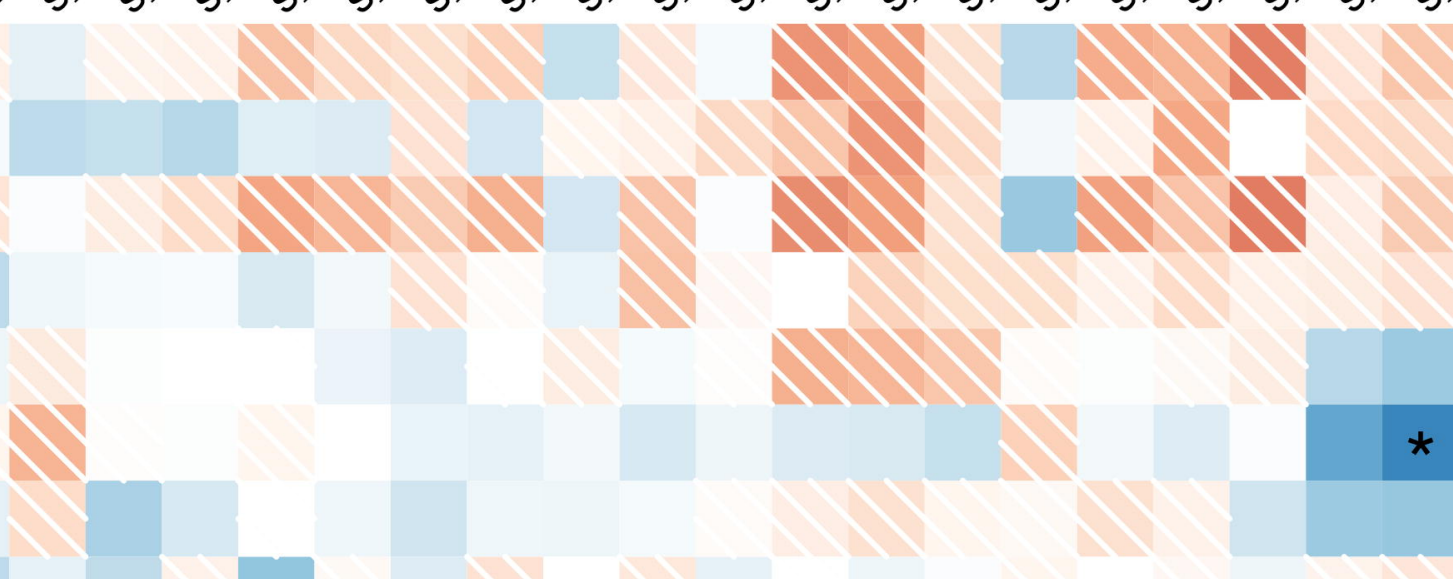

s_unknown_S24-7(ILLUMINA)

s_unknown_Dorea(ILLUMINA)

s_unknown_Parabacteroides(ILLUMINA)

S_unknown_Anaerovibrio(ILLUMINA)

s_unknown_Phascolarctobacterium(ILLUMINA)

S_Lactobacillus mucosae(ILLUMINA)

s_unknown_Prevotella(ILLUMINA)

S__Lactobacillus reuteri(ILLUMINA)

s_Prevotella copri(ILLUMINA)

s__Faecalibacterium prausnitzii(ILLUMINA)

S Prevotella stercorea(ILLUMINA

s_unknown_Lactobacillus(ILLUMINA)

s_unknown_[Prevotella](ILLUMINA)

s_unknown_Megasphaera(ILLUMINA)

s_unknown_[Ruminococcus](ILLUMINA)

s_unknown_Shuttleworthia(ILLUMINA) 


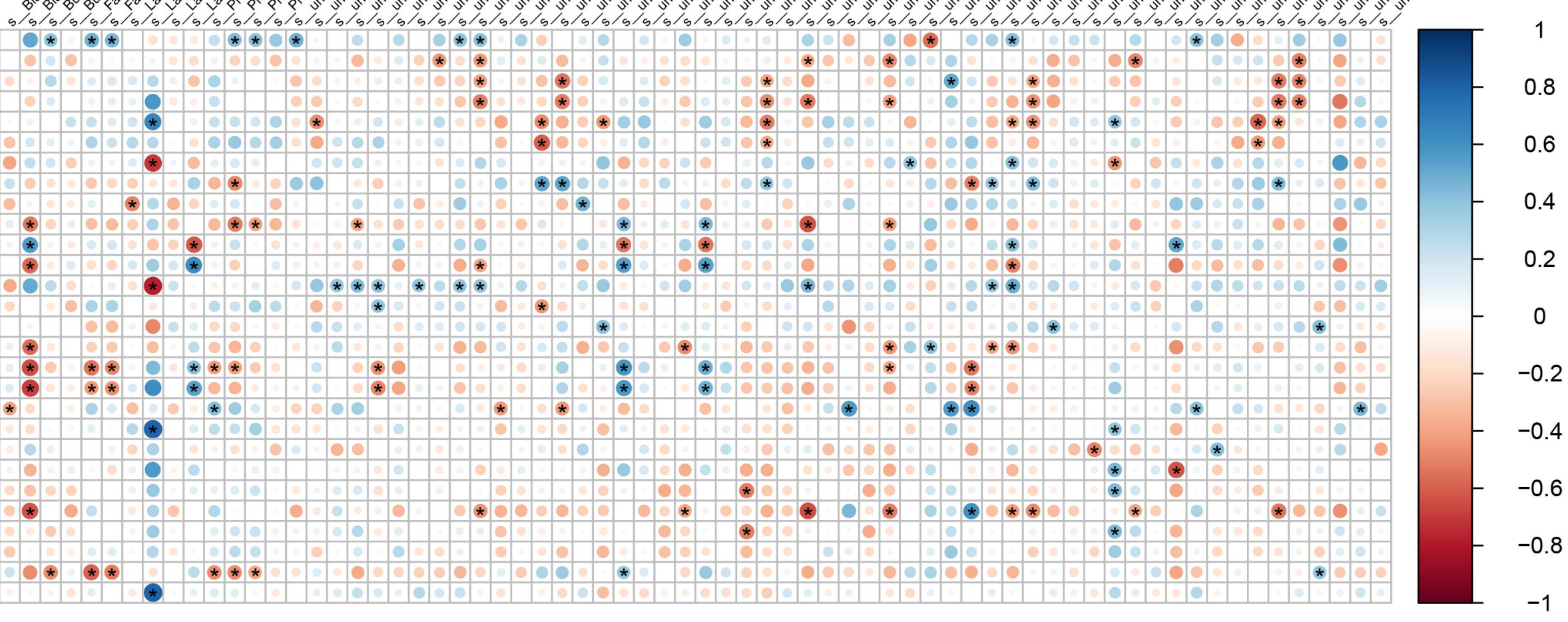

